\title{
Elevated arterial blood pressure after superior cavo-pulmonary anastomosis is associated with elevated pulmonary artery pressure and cerebrovascular dysautoregulation
}

\author{
Antonio G Cabrera ${ }^{1}$, Kathleen K Kibler ${ }^{2}$, R Blaine Easley ${ }^{2}$, Michelle Goldsworthy ${ }^{3}$, Lara S Shekerdemian ${ }^{3}$, \\ Dean B Andropoulos ${ }^{2}$, Jeffrey Heinle ${ }^{4}$, Erin A Gottlieb ${ }^{2}$, Eric $\mathrm{Vu}^{2}$ and Ken M Brady ${ }^{2}$
}

BACKGROUND: Elevated arterial blood pressure (ABP) is common after superior bidirectional cavopulmonary anastomosis (BCPA). The effects of elevated ABP after BCPA on cerebrovascular hemodynamics are unknown. We sought to determine the relationship between elevated $A B P$ and cerebrovascular autoregulation after BCPA.

METHODS: Prospective, observational study on infants with single-ventricle physiology after BCPA surgery. Continuous recordings of mean $A B P$, mean cavopulmonary artery pressure (PAP), near-infrared spectroscopy measures of cerebral oximetry (regional cerebral oxygen saturation $\left(\mathrm{rSO}_{2}\right)$ ), and relative cerebral blood volume index were obtained from admission to extubation. Autoregulation was measured as hemoglobin volume index $(H V x)$. Physiologic variables, including the $H V x$, were tested for variance across $A B P$.

RESULTS: Sixteen subjects were included in the study. Elevated ABP post-BCPA was associated with both, elevated PAP $(P<0.0001)$ and positive $\mathrm{HVx}$ (dysautoregulation; $P<0.0001)$. No association was observed between $A B P$ and alterations in $\mathrm{rSO}_{2}$. Using piecewise regression, the relationship of PAP to ABP demonstrated a breakpoint at $68 \mathrm{~mm} \mathrm{Hg}$ (interquartile range (IQR) $62-70 \mathrm{~mm} \mathrm{Hg}$ ). Curve fit of $\mathrm{HVX}$ as a function of $A B P$ identified optimal $A B P$ supporting robust autoregulation at a median ABP of $55 \mathrm{~mm} \mathrm{Hg}$ (IQR 51$64 \mathrm{~mm} \mathrm{Hg}$ ).

CONCLUSIONS: Elevated ABP post-BCPA is associated with cerebrovascular dysautoregulation, and elevated PAP. The effects, of prolonged dysautoregulation within this population, require further study.

$\mathbf{T}$ he bidirectional superior cavopulmonary anastomosis (BCPA) is performed as part of a staged palliation for patients born with single functional ventricle (1). During this surgery, the main pulmonary artery is oversewn when present, and the superior vena cava is transposed from the atria to the superior aspect of the branch pulmonary artery. The typical candidate for this surgery has a cyanotic, mixing lesion with parallel circulations to the pulmonary and systemic circuits, and lacks adequate ventricular substrate to septate the right from the left ventricle to create serial pulmonary and systemic circuits of circulation.

The BCPA results in a pulmonary circulation that is serial to the cephalad systemic circulation without an interposed ventricle. Pulmonary venous return mixes in the common atrium with systemic venous return from the inferior vena cava. The intended consequence of the BCPA is to improve hypoxia, reduce ventricular volume load, and provide a more stable source of pulmonary blood flow. An undesired consequence of the BCPA is to increase cephalad venous pressure. After the operation, superior cavopulmonary pressure is equivalent to pulmonary artery pressure (PAP) and becomes determinant of cerebral perfusion pressure. Postoperative elevation of arterial blood pressure (ABP) after this surgery is common, and there is a concern that lowering ABP will result in cerebral hypoperfusion when the cavopulmonary pressure is elevated (2). Furthermore, it has been speculated that elevated systemic ABP is necessary to maintain the transcerebral pressure gradient and therefore cerebral blood flow in the face of cerebral venous hypertension (3).

In this observational study, we sought to quantify the effect of ABP on cerebrovascular autoregulation after BCPA. Intact autoregulation during elevations of $\mathrm{ABP}$ and impaired autoregulation during normotension would imply that the elevated $\mathrm{ABP}$ is an adaptive response to maintain cerebral perfusion. Conversely, intact autoregulation during normotension and impaired autoregulation during elevations of $\mathrm{ABP}$ would imply that elevated ABP is a maladaptive response. We hypothesized an association between the state of cerebrovascular autoregulation and $\mathrm{ABP}$ such that elevated $\mathrm{ABP}$ is associated with dysautoregulation.

${ }^{1}$ Department of Pediatrics, Lillie Frank Abercrombie Section of Pediatric Cardiology, Baylor College of Medicine and Texas Children's Hospital, Houston, Texas; ${ }^{2}$ Department of Anesthesiology, Division of Pediatric Cardiovascular Anesthesiology, Baylor College of Medicine and Texas Children's Hospital, Houston, Texas; ${ }^{3}$ Department of Pediatrics, Section of Critical Care Medicine, Baylor College of Medicine and Texas Children's Hospital, Houston, Texas; ${ }^{4}$ Department of Surgery, Section of Congenital Heart Surgery. Baylor College of Medicine and Texas Children's Hospital, Houston, Texas. Correspondence: Antonio G. Cabrera (agcabrer@bcm.edu) 


\section{Brain autoregulation cavopulmonary shunt $\mid$ Articles}

\section{METHODS}

This study was approved by the institutional review board of the Baylor College of Medicine and was performed in the Cardiovascular Intensive Care Unit at Texas Children's Hospital. Informed consent was obtained for prospective data collection from the legal guardian of each enrolled subject during the preoperative surgical visit. All patients who undergo BCPA at Texas Children's Hospital have continuous monitoring of invasive ABP, invasive central venous pressure (with an inferior vena cava catheter), invasive PAP, and tissue oximetry (Invos, Covidien, Boulder, CO) of the right forehead and right flank during the first night after surgery, as part of routine clinical care.

\section{Study Protocol}

Data acquisition began after admission to the Cardiovascular Intensive Care Unit. Mechanical ventilation was provided via volume control mode at 10-12 ml/kg, $\mathrm{PEEP}=5 \mathrm{~cm} \mathrm{H}_{2} \mathrm{O} \mathrm{FiO}_{2}(0.3-0.6)$. Postoperative sedation and analgesia were provided with infusions of fentanyl (1-4 $\mu \mathrm{g} / \mathrm{kg} / \mathrm{min})$, dexmedetomidine $(0.3-1 \mu \mathrm{g} / \mathrm{kg} / \mathrm{h})$, and $/$ or midazolam $(0.05-0.3 \mathrm{mg} / \mathrm{kg} / \mathrm{h})$. During the study, all patients were receiving milrinone infusion $(0.25-0.75 \mu \mathrm{g} / \mathrm{kg} / \mathrm{min})$.

Physiologic parameters of ABP, PAP, and regional cerebral oxygen saturation $\left(\mathrm{rSO}_{2}\right)$ were recorded as digital outputs with a $240 \mathrm{~Hz}$ sampling rate from their respective monitors to a laptop computer using ICM plus software (Cambridge University, Cambridge, UK). The optical density of the $805 \mathrm{~nm}$ light wavelength (isosbestic to oxyand deoxyhemoglobin) in the INVOS monitor was recorded to the same file. Absorbance of light in this wavelength is inversely proportional to the density of hemoglobin in the cortical tissue being interrogated. Blood volume index (BVI) was rendered from the trended optical density of the $805 \mathrm{~nm}$ light as (1-Optical density). (4) For each subject, a single, synchronized, and time-stamped file of each recorded physiologic variable was created. Waveform analytics were performed using ICM plus software.

\section{Quantifying Cerebrovascular Pressure Autoregulation}

The hemoglobin volume index (HVx) has been described and validated previously (4). In brief, the HVx is a metric of pressure reactivity or pressure passivity, quantifying the timing of constriction and dilation of the cerebral vessels in response to low-frequency increases and decreases in ABP. BVI has been shown to accurately trend hemodynamic "slow waves" related to autoregulation and is a surrogate marker to gauge the timing of vascular constriction and dilatation in the HVx calculation.

Pressure autoregulation is a low-frequency physiologic response (5). Therefore, high-frequency oscillations and transients (pulse and respiratory variations) are first removed from the $\mathrm{ABP}$ and $\mathrm{BVI}$ recordings using consecutive10 second averages (6). A Pearson's correlation coefficient (the $\mathrm{HVx}$ ) quantifies the passivity of the BVI to ABP from 30 paired samples (300 s epochs). Although correlation is fundamentally a time-domain analysis, the low-pass filtering and $300 \mathrm{~s}$ sample buffer used for the HVx make it relatively specific for a frequency between 0.003 and $0.05 \mathrm{~Hz}$, which corresponds to the frequency of Lundberg's B waves, or "slow waves." These slow waves are found in both the ABP and a variety of intracerebral measurements and are commonly used for autoregulation monitoring (7). The HVx is repeated in overlapping time windows updated every $60 \mathrm{~s}$. A more positive HVx indicates that BVI is more passive to $\mathrm{ABP}$, a state of impaired autoregulation. A more negative $\mathrm{HVx}$ indicates that $\mathrm{BVI}$ is more reactive to $\mathrm{ABP}$, a state of intact autoregulation.

\section{Identifying Optimal ABP for Pressure Autoregulation}

$\mathrm{HVx}$ values were binned and averaged according to the ABP at which they were measured in $5 \mathrm{~mm} \mathrm{Hg}$ increments (8). The curve-fit algorithm developed and previously described by Aries et al. (9) was applied to this binned data using the entire recording session to render a single optimal ABP. Optimal ABP identified by this algorithm is conceptualized as the ABP at which HVx is lowest, with consistently ascending values on either side. The curve fit is performed on binned, averaged data to smooth the signal noise that results when measuring pressure autoregulation from spontaneous slow waves of ABP. The performance of the algorithm is dependent on the spontaneous range of $\mathrm{ABP}$ experienced by the subject, as both intact and impaired ranges of $\mathrm{ABP}$ must be measured for the algorithm to render an optimal value. Inadequate recordings render a non-numerical value when the algorithm is used.

\section{Statistics}

Patient characteristics and study variables are presented as median and interquartile range for the cohort. Continuous variables are summarized as mean and standard deviation for individual patient recordings. Associations between the study variables $\left(\mathrm{HVx}, \mathrm{rSO}_{2}\right.$, and PAP) and $\mathrm{ABP}$ were tested with accounting for highly repetitive samples that were inconsistently experienced across the range of $A B P$ studied. To do so, each study variable was first binned according to the $\mathrm{ABP}$ at which it was observed in $5 \mathrm{~mm} \mathrm{Hg}$ increments for each individual subject. Bins containing $<1 \%$ of the total data for an individual subject were excluded from the analysis as an artifact filter. The individual subject bins were then aggregated to a cohort binning as a mean of means. This binning and averaging process equalizes the contribution of each subject to final analysis, regardless of variability in the duration of recording. However, not every subject experienced every $\mathrm{ABP}$, so the inconsistently repetitive measures were analyzed for variance across $\mathrm{ABP}$ using linear regression with generalized estimation of equations (STATA, College Station, TX) (10).

The study variables $\mathrm{HVx}$ and $\mathrm{rSO}_{2}$ were analyzed for variance across PAP with the same binning, averaging, and analysis method described above for ABP. Given the smaller range of PAP observed, $2 \mathrm{~mm} \mathrm{Hg}$ bins of PAP were chosen.

A nonlinear PAP-ABP relationship was observed in individual recordings. At lower $\mathrm{ABP}, \mathrm{PAP}$ was correlated to $\mathrm{ABP}$ with a small positive slope. At higher $\mathrm{ABP}$, the $\triangle \mathrm{PAP} / \triangle \mathrm{ABP}$ slope was noticeably larger. Piecewise regression from 1-min averaged data was used to identify the cutoff ABP for each subject's data set above which the $\Delta \mathrm{PAP} / \Delta \mathrm{ABP}$ was increased.

\section{RESULTS}

Thirty eligible subjects were identified between October 2014 and September 2015 and data are presented for 16 subjects. Reasons for dropout included consent denial (four subjects), multiple eligible subjects on the same day with the lack of available equipment/personnel to collect the data (seven subjects), and recording failure (three subjects). All recorded data were analyzed and included. The median duration of the recording was $18 \mathrm{~h}(8-20 \mathrm{~h})$. Preoperative and intraoperative subject characteristics are presented in Table 1. Age and weight were 155 days (113-198) and $6.2 \mathrm{~kg}$ (5.5-7.5), respectively, with a body surface area equal to $0.33 \mathrm{~m}^{2}$ (0.31-0.37). There were 11 patients with a morphologic right ventricle, 5 with a morphologic left ventricle. Nine patients had undergone aortic arch reconstruction as part of a stage I palliation procedure. Median cardiopulmonary bypass and cross clamp times were $131 \mathrm{~min}$ (97-174) and $9.5 \mathrm{~min}(0-46)$, respectively. Physiologic variables from the monitoring period are shown for each subject in Table 2 .

Figure 1 summarizes the analyses of study variable associations with ABP.

The study variables $\mathrm{HVx}$ and PAP were both significantly variant across $\mathrm{ABP}(P<0.0001$ for both associations), but $\mathrm{rSO}_{2}$ was not associated with ABP $(P=0.07)$. Intact 
Articles | Cabrera et al.

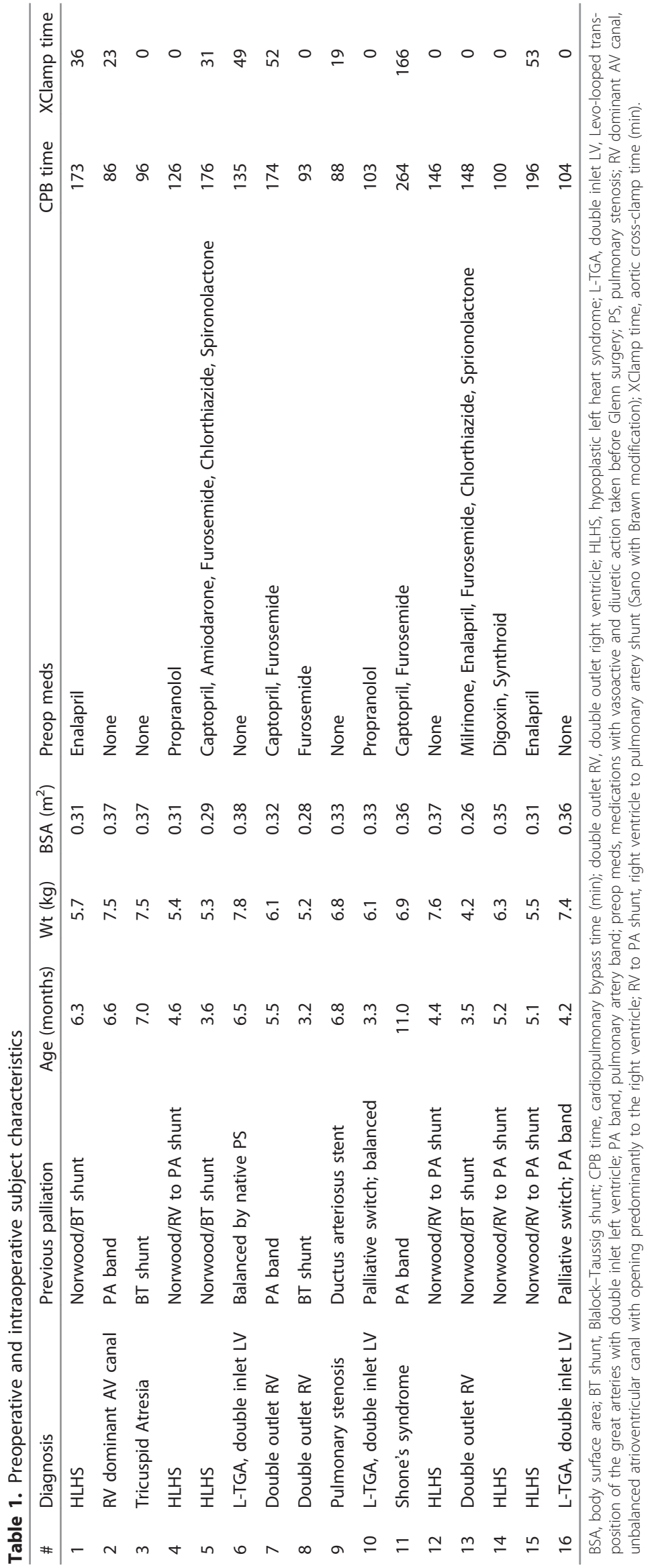




\section{Brain autoregulation cavopulmonary shunt Articles}

Table 2. Physiologic data averaged over the monitoring period of the study

\begin{tabular}{|c|c|c|c|c|c|c|c|c|}
\hline No. & $\mathrm{ABP}$ & PAP & CVP & $\mathrm{P}_{\mathrm{a}} \mathrm{CO}_{2}$ & $\mathrm{rSO}_{2}$ cerebral & $\mathrm{rSO}_{2}$ somatic & $\mathrm{ABP}_{\mathrm{OPT}}$ & $\triangle \mathrm{PAP} / \triangle \mathrm{ABP}$ breakpoin \\
\hline 1 & $59 \pm 6$ & $12 \pm 4$ & $6 \pm 3$ & 41 (39-44) & $58 \pm 2$ & $83 \pm 4$ & 52 & 60 \\
\hline 2 & $70 \pm 11$ & $16 \pm 8$ & $9 \pm 4$ & 37 (36-39) & $59 \pm 6$ & $82 \pm 6$ & 64 & 74 \\
\hline 4 & $63 \pm 7$ & $13 \pm 3$ & $9 \pm 10$ & $38(37-41)$ & $49 \pm 7$ & $78 \pm 5$ & nan & nan \\
\hline 5 & $70 \pm 10$ & $15 \pm 4$ & $9 \pm 4$ & $45.5(44-46)$ & $54 \pm 7$ & $92 \pm 3$ & 65 & 70 \\
\hline 8 & $66 \pm 13$ & $15 \pm 1$ & $8 \pm 3$ & $44.5(37-53)$ & $33 \pm 4$ & $78 \pm 1$ & nan & nan \\
\hline 9 & $69 \pm 6$ & $15 \pm 2$ & $10 \pm 2$ & $42(40-43)$ & $65 \pm 4$ & $74 \pm 6$ & 50 & 62 \\
\hline 10 & $58 \pm 15$ & $9 \pm 7$ & $10 \pm 8$ & $49(40-54)$ & $47 \pm 5$ & $73 \pm 7$ & 55 & 70 \\
\hline 11 & $61 \pm 10$ & $13 \pm 8$ & $9 \pm 4$ & $43.5(40-47)$ & $49 \pm 11$ & $82 \pm 4$ & 50 & 63 \\
\hline 15 & $77 \pm 12$ & $14 \pm 9$ & $8 \pm 6$ & $37(35-38)$ & $51 \pm 5$ & $83 \pm 5$ & 65 & 66 \\
\hline 16 & $65 \pm 9$ & $13 \pm 4$ & $10 \pm 9$ & $40(35-45)$ & $53 \pm 6$ & $80 \pm 9$ & 62 & 60 \\
\hline
\end{tabular}

$\mathrm{ABP}$, mean arterial blood pressure $\pm \mathrm{SD}(\mathrm{mm} \mathrm{Hg}) ; \mathrm{ABP}_{\mathrm{OPT}}$, arterial blood pressure at which autoregulation is optimized using the algorithm referenced in Methods ( $\mathrm{mm}$ Hg); $\mathrm{CVP}$, central venous pressure measured from inferior vena cava catheter $\pm \mathrm{SD}(\mathrm{mm} \mathrm{Hg}) ; \triangle P A P / \triangle A B P$ breakpoint, arterial blood pressure at which the slope of the PAP-ABP relationship inflects, determined by piecewise regression $(\mathrm{mm} \mathrm{Hg})$; nan, non-number returned by the curve-fit analysis; $P_{a} \mathrm{CO}_{2}$, median arterial carbon dioxide tension (IQR); PAP, pulmonary artery pressure measured from cavopulmonary shunt catheter $\pm \mathrm{SD}(\mathrm{mm} \mathrm{Hg}) ; \mathrm{rSO}_{2}$ cerebral, regional tissue oximetry measured over the right forehead $\pm \mathrm{SD}$ (\% saturation); $\mathrm{rSO}_{2}$ somatic, regional tissue oximetry measured over the right flank $\pm \mathrm{SD}$ (\% saturation).

autoregulation and low PAP were observed during normotension. By contrast, dysautoregulation and elevated PAP were observed during elevations of ABP.

The increase of PAP with increases in $\mathrm{ABP}$ was not linear (Figure 1b). There was an observed sustained state of elevated $\mathrm{ABP}$, elevated $\mathrm{PAP}$, and dysautoregulation in $58 \%$ of the aggregated recordings (Figure 1d). Owing to the nonlinear nature of these associations, we determined the natural demarcation of each individual recording where the $\triangle \mathrm{PAP} /$ $\triangle \mathrm{ABP}$ slope increased with piecewise regression. This inflection occurred at a median ABP of $68 \mathrm{~mm} \mathrm{Hg}(62-$ $70 \mathrm{~mm} \mathrm{Hg}$ ). In other words, during normotension (mean ABP $<68)$ for this cohort, the $\triangle \mathrm{PAP} / \triangle \mathrm{ABP}$ slope was $0.17(0.05-$ 0.23 ) and during elevated $\mathrm{ABP}$ (mean $\mathrm{ABP}>68$ ), the $\triangle \mathrm{PAP} /$ $\triangle \mathrm{ABP}$ slope was $0.68(0.5-1.0)$, which is a fourfold increase in slope $(P<0.001)$.

A striking feature of the autoregulation analysis of this data set is that no apparent lower limit of autoregulation (hypotension associated with dysautoregulation) was observed in any subject. However, dysautoregulation associated with elevated $\mathrm{ABP}$ was frequently observed and was significant $(P<0.0001$, Figure 1c). The median ABP at which HVx optimized was $55 \mathrm{~mm} \mathrm{Hg}(51-64 \mathrm{~mm} \mathrm{Hg})$.

The visual juxtaposition of Figure $\mathbf{1 b}, \mathbf{c}$ is suggestive that there is an association between the states of elevated PAP and dysautoregulation, as they occur at similar thresholds of ABP elevation. Dysautoregulation was tested and observed to be a function of PAP such that elevated PAP was associated with a significant increase in $\operatorname{HVx}(P<0.0001$, Figure 2). Therefore, the association between elevated $\mathrm{ABP}$, dysautoregulation, and elevated PAP cannot be evaluated without accounting for the collinearities of these three variables.

\section{DISCUSSION}

This study is a prospective observation of the relationship between standard ABP management after BCPA surgery and cerebrovascular autoregulation. We hypothesized a finding of impaired autoregulation, worsening with elevated ABP. We did find that elevated systemic ABP is associated with dysautoregulation, but with a strong colinearity between the three study variables: elevated ABP was associated with both dysautoregulation $(\mathrm{HVx})$, and superior vena cava venous pressure (PAP).

Based on the study design, the statistics used describe common trends within the cohort, rather than differences between subjects with different physiology. We observed that patients within this study transitioned between two states: one with normotension, low PAP, and intact autoregulation, and another with elevated ABP, elevated PAP, and impaired autoregulation. The delineation of "elevated $\mathrm{ABP}$ " from "normotension" is performed from the analysis of cerebrovascular autoregulation in the cohort (Figure 1), not imported from normative data. Our study does not show the primary driver of transition between these two states, nor does it show the effect of elevated ABP, elevated PAP, or dysautoregulation on outcome. Although normative data on ABP in infants without cardiac disease from 0 to 6 months has been reported to range between 60 and $65 \mathrm{~mm} \mathrm{Hg}$, the specific range for the BCPA population is unknown (11). 

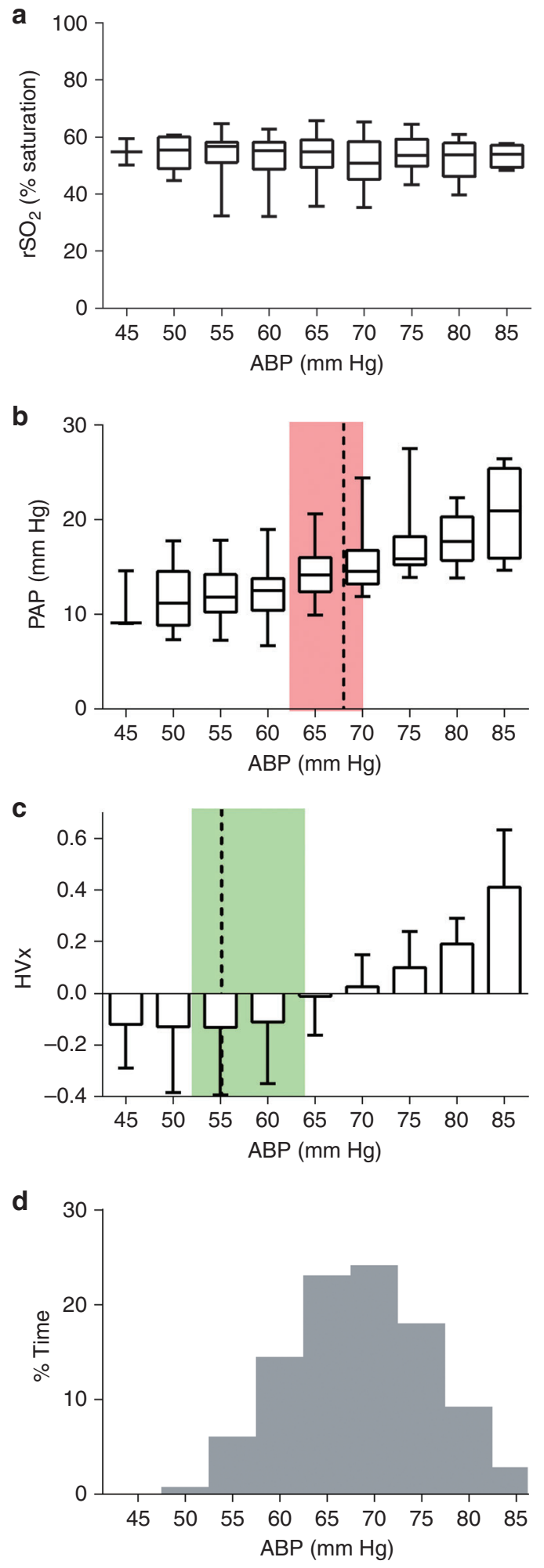

Our data are consistent with previous studies of antihypertensives after BCPA. Lee et al. (12) performed catheterization and magnetic resonance imaging on an older cohort of children (median 28 months) with cavopulmonary shunt during pre-Fontan evaluation. These subjects were given intravenous enalaprilat to reduce $\mathrm{ABP}$ from a median of
Figure 1. High arterial blood pressure (ABP) after Glenn shunt is associated with elevated pulmonary artery pressure (PAP) and dysautoregulation. (a) Cerebral oximetry $\left(\mathrm{rSO}_{2}\right)$ was not associated with $A B P$ in this cohort. (b) Systemic hypertension was associated with elevated PAP $(P<0.0001)$. A nonlinear increase in PAP with increase in $A B P$ was observed. Individual subject cutoffs for increased $\triangle P A P / \triangle A B P$ are shown with a vertical dashed line and vertical red shading at $68 \mathrm{~mm} \mathrm{Hg}(62-70 \mathrm{~mm} \mathrm{Hg})$. (c) Hypertension was associated with increased hemoglobin volume index ( $H V x, P<0.0001)$, signifying dysautoregulation. Individual subject optimal ABP for autoregulation are shown with a vertical dashed line and green shading at $55 \mathrm{~mm} \mathrm{Hg}(51-$ $64 \mathrm{~mm} \mathrm{Hg}$ ). (d) The percent time at each ABP for the aggregate recordings are presented.
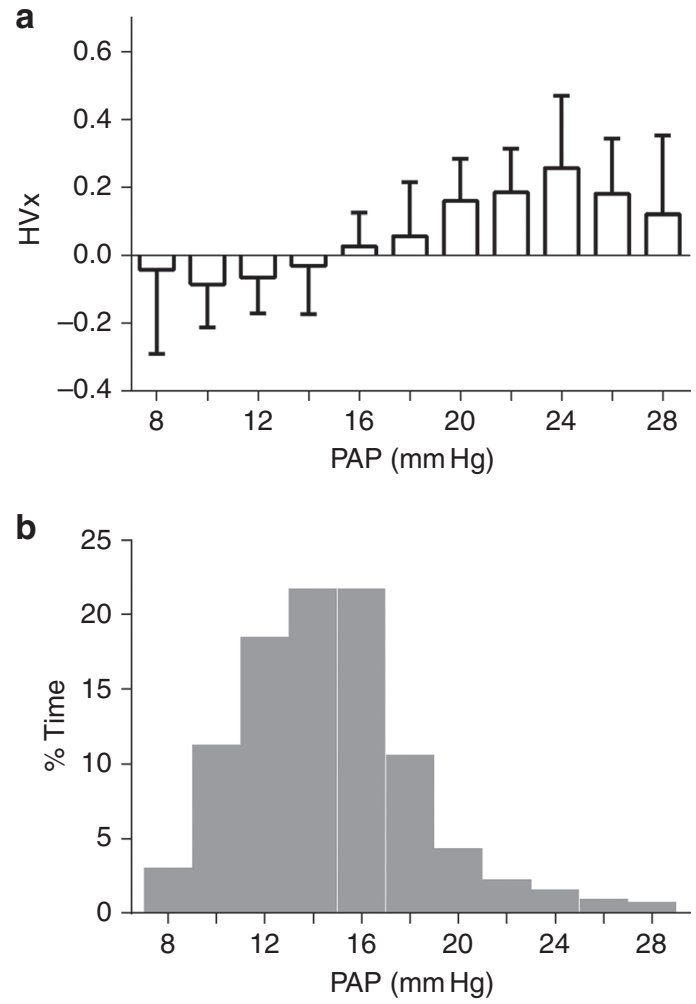

Figure 2. Elevated cavopulmonary pressure (PAP) is associated with dysautoregulation. (a) The hemoglobin volume index $(\mathrm{HVX})$ is positive, indicating impaired autoregulation when the PAP is elevated; $\mathrm{HVx}$ is negative, indicating intact autoregulation when PAP is lower. (b) The percent time at each increment of PAP is shown from the aggregate data.

$62 \mathrm{~mm} \mathrm{Hg}$ to a median of $42 \mathrm{~mm} \mathrm{Hg}$. Cardiac output was unchanged, but redistributed to the caudad circulation with minimal decrease in cerebral blood flow. Their data suggest that pressure autoregulation is present during normotension and mild hypotension in the setting of cavopulmonary shunt. Our measurement of pressure reactivity in immediate postoperative cavopulmonary shunt patients also shows intact pressure autoregulation during normotension.

In a separate, prospective, controlled study of nine subjects, Simsic et al. (3) reported on the use of sodium nitropruside 


\section{Brain autoregulation cavopulmonary shunt Articles}

infusion after BCPA. After a $20 \%$ reduction in ABP (from $69 \pm 6$ to $58 \pm 6 \mathrm{~mm} \mathrm{Hg}$ ), there was no significant change of middle cerebral artery flow velocity and no change in the arteriovenous oxygen saturation difference across the cephalad circulation, suggesting intact cerebrovascular autoregulation at normal ABP for age.

Both the Pejovic et al. (11) and Simsic et al. (3) studies and the present study suggest that it is safe to treat elevated $A B P$ after $\mathrm{BCPA}$, and that normal $\mathrm{APB}$ restores intact cerebral autoregulation. The common concern that elevated PAP necessitates a higher ABP to maintain cerebral perfusion pressure is not supported by the available data. Questions raised by these studies and the data presented here are whether that treatment can result in transition from the dysautoregulated state to the autoregulated state, whether that treatment is best aimed to lower PAP (with diuretics or pulmonary vasodilators) or ABP (with antihypertensive drugs), and finally whether maintaining a state of normotension, low PAP, and intact autoregulation results in any improvement of measureable short- or long-term outcome.

The specific cause of elevated systemic ABP after BCPA is unknown. Hjortdal et al. (13) found that there is a significant neurohormonal activation late after cavopulmonary anastomosis operations. They showed that angiotensin II, renin, and aldosterone levels were significantly increased, and that there was a twofold increase in vasopressin, atrial natriuretic factor, and brain natriuretic peptide compared with healthy controls. Interestingly, they did not find associations between the neurohormonal activation and hemodynamic variables. Characterization of neurohormonal activation before and after the BCPA may be useful to delineate the mechanism and treatment options for postoperative hypertension in these subjects.

The main limitation of this retrospective study is the small sample size. Further, lack of a controlled intervention on ABP prevents discussion of causality for the findings. All subjects were on dexmedetomidine infusions during the recordings. While this $\alpha-2$ adrenergic agonist may have an attenuating effect on output from the sympathetic chain ganglion, the subjects on dexmedetomidine in this study were not prevented from experiencing hypertension by this sedative.

In summary, this study found that elevated $\mathrm{ABP}$ after BCPA is associated with elevated cavopulmonary pressure and a state of cerebrovascular dysautoregulation. Maintenance of optimized $\mathrm{ABP}$ in the postoperative period after $\mathrm{BCPA}$ is associated with intact cerebrovascular pressure autoregulation and lower cavopulmonary pressure. Future studies to explore the mechanism of impaired pressure autoregulation and elevated $\mathrm{ABP}$ in this population is warranted, as well as evaluating how these observations are related to acute and long-term neurologic outcomes.

\section{STATEMENT OF FINANCIAL SUPPORT:}

This work was supported by institutional funds at Texas Children's Hospital.

Disclosure: The authors declare no conflict of interest.

\section{REFERENCES}

1. Glenn WW. Superior vena cava-pulmonary artery anastomosis. Ann Thorac Surg 1984;37:9-11.

2. Chang AC, Hanley FL, Wernovsky G, et al. Early bidirectional cavopulmonary shunt in young infants. Postoperative course and early results. Circulation 1993;88 (Part 2): II149-58.

3. Simsic JM, Bradley SM, Mulvihill DM. Sodium nitroprusside infusion after bidirectional superior cavopulmonary connection: preserved cerebral blood flow velocity and systemic oxygenation. J Thorac Cardiovasc Surg 2003;126:186-90.

4. Lee JK, Kibler KK, Benni PB, et al. Cerebrovascular reactivity measured by near-infrared spectroscopy. Stroke 2009;40:1820-6.

5. Fraser CD 3rd, Brady KM, Rhee CJ, et al. The frequency response of cerebral autoregulation. J Appl Physiol 2013;115:52-6.

6. Czosnyka M, Smielewski P, Kirkpatrick P, Menon DK, Pickard JD. Monitoring of cerebral autoregulation in head-injured patients. Stroke 1996;27:1829-34.

7. Czosnyka M, Brady K, Reinhard M, Smielewski P, Steiner LA. Monitoring of cerebrovascular autoregulation: facts, myths, and missing links. Neurocrit Care 2009;10:373-86.

8. Steiner LA, Czosnyka M, Piechnik SK, et al. Continuous monitoring of cerebrovascular pressure reactivity allows determination of optimal cerebral perfusion pressure in patients with traumatic brain injury. Crit Care Med 2002;30:733-8.

9. Aries MJ, Czosnyka M, Budohoski KP, et al. Continuous determination of optimal cerebral perfusion pressure in traumatic brain injury. Crit Care Med 2012;40:2456-63.

10. Zeger SL, Liang KY. Longitudinal data analysis for discrete and continuous outcomes. Biometrics 1986;42:121-30.

11. Pejovic B., Peco-Antic A., Marinkovic-Eric J. Blood pressure in noncritically ill preterm and full-term neonates. Pediatr Nephrol 2007;22:249.

12. Lee KJ, Yoo SJ, Holtby $\mathrm{H}$, et al. Acute effects of the ACE inhibitor enalaprilat on the pulmonary, cerebral and systemic blood flow and resistance after the bidirectional cavopulmonary connection. Heart 2011;97:1343-8.

13. Hjortdal VE, Stenbog EV, Ravn HB, et al. Neurohormonal activation late after cavopulmonary connection. Heart 2000;83:439-43. 